main a considerable residuum suffering from cardiac, renal, or lung troubles, acquired or inherited blood taints, \&c., requiring almost continuous observation and treatment during the whole period. This broad and, as I venture to submit, proper view as to one's relation with one's patients at this crisis of their lives would be fruitful in the prevention of an incalculable amount not only of maternal, but also of infantile ill-health and mortality.

Secondary only in importance to the satisfying oneself as to the possession of needful strength on the part of one's patient to undergo the ordeal of gestation and parturition is the control of the selection of the nurse, upon whose obedience and intelligence the issue of a labour case largely dependsthe necessity for this control being greater in first cases than in others, inasmuch as the young and inexperienced mother is as a rule plastic in the hands of those who are about her; and that she very frequently suffers from this trustfulness is attested by the very large proportion of pelvic troubles which date from first confinements. The custom which now largely prevails of the patients choosing their own nurses is mainly responsible for a great deal of puerperal disease and death, and that this is so my second case illustrates very markedly. A very large number of women, more especially in country districts, take to munthly nursing without any special qualification or training for the task, and without the most elementary notions of the hygienic requirements either of the lyingin chamber or the lying-in patient; and, unfortunately for the medical profession at large, the harm which these women often do is never found out till it is too late, whilst the blame is frequently wrongly laid at the doctor's door. Did medical men only make it an essential part of the contract that they should have the selection of the nurse, and satisfy themselves not only of her capability, but also of her freedom from the danger of infection, puerperal accidents would undergo a very substantial diminution. Closely connected with the matter of the nurse is also, when the social position of the patient will allow it, the choice of the lying-in chamber. This ought clearly to be as carefully chosen as if a surgical operation had to be undergone. Especially should a badly lit, ill-ventilated room be avoided, or one where, by reason of proximity to a watercloset or bath-room with suspicious sanitary arrangements, danger to the patient is likely to follow. The satisfactory recovery of the patient after parturition, it must never be forgotten, depends largely apon her environment.

Finally, instead of the visits terminating at the eighth or tenth day after labour, they ought to be spread - over the whole period of normal uterine involutionthat is to say, from four to six weeks; in fact, the patient ought not to be left until local examination has proved that a satisfactory recovery has been made. The present mode of limiting the after-visits to four or five, or it may be six, leaving the patient's future actions to be regulated by her feelings or the advice-good or otherwiseof those who are about her, is largely responsible for much ill bealth. No uterine trouble is more common in our hospitals than subinvolution, nor one which so clearly has for its cause premature getting up of the parturient woman, and with which there are others, though less common, which might be equally well avoided by the medical attendant insisting upon the needful time being given to the parts to recover from the damage necessary by the Birth of the child. In opposition to what has thus been somewhat imperfectly sketched out, the medical profession may with justice say that the remuneration which now is occasionally grudgingly paid by the public, and is in a large number of instances, to my mind, totally inadequate to the responsibility and the time spent, renders the scheme impracticable; but to this I may be allowed to answer that, although I have carefully refrained from touching upon the matter of the monetary equivalent for such services, they have not been forgotten by me, and that the time has now arrived when such a scheme, or some modification of $i b$, should be adopted by the medical profession at large. My own feeling is that instead of the present system a fee, according to the posirtion of the patient, should be charged for attendance upon the confinement itself, and that attendances before and after this date should be charged as ordinary visits. In conclusion, I hope that at least this paper will draw the attention of the profession to a question which is of grave and every.day importance.

Nottingham.

\section{Clinital hlotes:}

\section{MEDICAL, SURGICAL, OBSTETRICAL, AND THERAPEUTICAL.}

\section{NOTES ON A SERIES OF CASES OF INFLUENZA.} By William Stanwell, M.R.C.S.

IN the belief that small contributions, even if premature, may be of some service, I forward the notes of fifty-three cases of influenza that I have had under treatment from the 8th of April to the 11th of May (fifteen in April, thirty-eight in May). The incubation period seems to be from three to seven days. This conclusion is derived from a study of the following facts:-1. Cases on the following dates all had opportunities of transmitting to one another, April 8th, 11th, 15th, 23rd, 24th, 29th, May 2nd, 2nd, 5th. 2. Where more cases than one have occurred in one house on different days, the following are dates of occurrence :-

$$
\begin{gathered}
\text { House A.-April 15th, 23rd, 27th, 27th, May 8th. } \\
\text { " } \quad \text { B.-May 1st, 1st, 7th. } \\
\text { " } \\
\text { C.-May 2nd, 5th, 5th, 9th. } \\
\text { D.-May 3rd, 3rd, 3rd, 11th. } \\
\text { " E.-May 4th, 11th. } \\
\text { " F.-May 5th, 10th. } \\
\text { G.-May 8th, 11th, 11th, 11th. }
\end{gathered}
$$

In B. case on May 7th and in F. case on May 10th had no opportunity of contracting elsewhere, an incubation of five days in one, six days in the other. In $\mathrm{C}$. there is very little chance of a second contagion from without.

The invasion is marked by chilliness, shivering, feeling of cold water down the back, occasionally rigor, followed by a rapid rise of temperature to $1025^{\circ}$ or $103.5^{\circ}$ (highest I have taken $105 \cdot 1^{\circ}$, with intense headache, aching in back and limbs of more or less severity, suffused eyes, varying degree of sneezing and coryza, sometimes none; laryngeal sore-throat with hoarseness and cough, secretion as a rule very scanty; in some cases vomiting; natural emesis seems beneficial. In the majority of cases the temperature falls in from twenty-four to thirty-six hours to normal, the fall being accompanied as a rule by profuse perspiration; with this fall of temperature the various aches very rapidly disappear, and a varying amount of prostration and debility remains for a short time. The appetite for food, which has been at zero, rapidly rises, and in from six to ten days in uncomplicated cases the patient is well. In fifty-three cases I have had two cases of pneumonia, one very severe ; and one case in which twelve days after the first attack a relapse or second attack supervened, in every way identical with the first. As yet $I$ have had no fatal cases. In several cases I have seen during convalescence a tendency to vertigo, and in a large number of cases slow pulse (40 to 50) occurs, and lasts for some days after the fall of temperature.

Treatment.-Bed, with abundance of covering, to encourage free perspiration, which appears to be desirable. Five grains of sulphate of quinine in powder in the fever stage brings down the temperature more quickly than is the case where no antipyretic is given; this seems to be of importance, as the intense pains disappear with the lowering of the temperature, and the curtailing of the pains is the consummation most devoutly wished for by the sufferer Afterwards I prescribe tincture of Indian hemp, with or with out iodide of potassium, and the tinctures of barkandrhubarb, or the tincture of bark with acids. In the present epidemic, as in that of last year, I have found the tincture of Indian hemp of the greatest value in almost all cases, bub more particularly in the more debilitated cases, with slow pulse, and very little, if any, inclination for food or energy. Diet: Alcohol and beef-tea I forbid, as they seem to aggravate; milk and soda-water, tea and toast, and light gruel are all that is desired for the first twenty-four hours, nor does it seem necessary to press food; afterwards, nnder judicious treat. ment, the appetite soon returns, food is relished, and convalescence complete.

Rochdale. 